\section{Locus swarm}

\section{By Steve Edelson, Executive Editor}

Although whole-genome association studies in the autoimmune space are increasingly common, their predictive value is still limited. The main reason is that autoimmune diseases have multiple genetic causes, and any one risk factor will only contribute slightly to the disease phenotype.

Nevertheless, pieces of the puzzle are being assembled and companies developing autoimmune therapeutics are watching closely as new loci are published. With enough loci, companies expect to be able to triangulate the findings in order to potentially make therapeutic development decisions.

An example of such triangulation is the identification of a risk locus involved in multiple autoimmune diseases by researchers from University Medical Center Utrecht. The work was published in The American Journal of Human Genetics. ${ }^{1}$

\section{"We cluster these publications to build a case."} Genmab A/S
-Jan van de Winkel,

In the autoimmune space, Koeleman thinks researchers have found about $10 \%$ of the risk loci. "If the pace goes like it's been going, we should have most of them in 5-10 years. We used to type a single SNP by hand in a week or so. Now we can do 1 million in a week."

Cisca Wijmenga, an author on the paper and head of the Complex Genetics section in the Department of Medical Genetics at Utrecht, said there are two main ways to approach the task of finding additional risk loci. "One way is to look at a marker for a specific disease and see if it applies elsewhere," which is what their group did. The alternative method is to "look at the pathway the gene fits in and look for other genes in the pathway."

She added: "With association studies, you're not pinpointing the variants that cause a disease. Rather, you're pinpointing a region in the genome" that likely contributes to disease, but may not be fully responsible for the disease phenotype.

The challenge with association studies, according to Wijmenga, is that the functional work that follows is time consuming.

The locus on chromosome 4q27 contains four genes: KIAA1109, Tenr, interleukin-2 (IL-2) and IL-21. Wijmenga expects that variants in the latter two are the likely contributors to autoimmune diseases.

"I don't expect the other two to be important," she said. "The Tenr gene is expressed only in the testes, so it's hard to make a story for that. The KIAA1109 gene has an unknown function. For $I L-2$ and $I L-21$, we already know they're associated with autoimmune disease."

The Dutch researchers sought to confirm and build on two recent genome-wide studies. The first study found a 480-kb block on chromosome 4q27 containing SNPs involved in celiac disease; ${ }^{2}$ the second identified the same block's association with type 1 diabetes. ${ }^{3}$

Based on these results, the Utrecht group decided to take the most common SNP identified in the celiac study and see whether it also appeared in an independent cohort of 350 type 1 diabetes patients. They also looked at another autoimmune disease-rheumatoid arthritis (RA) - by analyzing two RA cohorts with a total of 1,047 patients. Compared with a group of 929 controls, the celiac disease SNP variant was significantly associated with both type 1 diabetes and RA ( $p=0.0006$ and $p=0.0002$, respectively).

According to Bobby Koeleman, a professor in the university's Department of Medical Genetics and an author on the paper, the finding has two main implications. "The first is that the association is true. All genome-wide results suffer from a false-positive finding rate. But if we can replicate a study, it's probably a true risk locus. And because we replicated it in different autoimmune diseases, it's an implication that we have a general autoimmune locus."

The challenge, said Koeleman, is that "all these new risk factors confer very low risk." For example, he said people with the locus on chromosome 4q27 likely have "a 1.2-fold higher risk than someone who does not have the association. It's not like breast cancer, where there's a 30-50-fold higher risk [for patients with BRCA gene mutations] or Huntington's where if you have the mutation you will get the disease."
Both IL-2 and IL-21 are type 1 cytokines with multiple roles in stimulating T cells. $I L-2$ has previously been shown to be a susceptibility gene in a mouse model of type 1 diabetes. ${ }^{4}$

The risk locus paper adds to the body of work that provides scientific support for the HuMax-TAC program at Genmab A/S. The antibody targets the IL-2 receptor $\alpha$-chain (CD25) TAC antigen, which is overexpressed by activated T cells. It is in preclinical development to treat autoimmune, inflammatory and hyperproliferative skin disorders, as well as acute transplant rejection.

Genmab, an antibody company focused on cancer and autoimmune diseases, is keeping a close eye on risk-association studies that emerge from academia. EVP and CSO Jan van de Winkel said the company collects data like those published in the American Journal of Human Genetics paper.

The paper, said van de Winkel, "suggests that IL-2 may be relevant in other autoimmune diseases, and that helps us think about positioning and maybe pilot studies. We cluster these publications to build a case. We won't start a program on the basis of one paper. Rather, it's recent data from multiple studies."

Genmab's therapeutic development decisions in some cases involve triangulating data, including risk locus studies, to pick the most relevant diseases.

For example, Genmab first developed HuMax-TAC for transplant rejection. Over time, said van de Winkel, "there were data pointing to 
other autoimmune indications. From the time we made the antibody, the published data grew stronger and stronger. Factors like IL-2 play an important role in keeping the inflammation going. I don't think it's the single reason for autoimmune diseases-there are probably many other genes-but it does contribute to the phenomenon."

Indeed, whether IL-2 actually is a root cause of autoimmune disease is almost beside the point, said van de Winkel. The key, he said, is that the IL-2 pathway is another avenue of attack.

Targeting a variety of pathways is necessary because "not all the same pathways are in the same patients," van de Winkel said. "The [tumor necrosis factor] pathway, for example, is involved in only about half of rheumatoid arthritis patients. You need access to a number of targeted therapies to control the disease going forward."

Although therapeutic strategies will clearly emerge from the discovery of new risk loci, both Wijmenga and Koeleman noted that completing the full genetic puzzle of many diseases will be hampered by rare gene variants.

"If you find one variant in one patient, what does it mean?" said Wijmenga. "It could be causing the disease or it could just be there by coincidence. You'd have to do functional studies to see whether it affects the function of the protein in some way."

Biopharma companies, however, are not going to wait for the identification of a complete set of risk factors before making therapeutic development decisions. A case in point is Tolerx Inc., which is going after a different diabetes target.

Otelixizumab, an anti-CD3 mAb, is expected to start Phase III testing to treat type 1 diabetes in 1Q08. The company also plans to start a clinical trial this year with this antibody to prevent type 1 diabetes in patients with a different set of undisclosed genetic markers identified via association studies. The compound is partnered with GlaxoSmithKline plc.

Tolerx president and CEO Douglas Ringler thinks that preventing type 1 diabetes might be easier than treating it, because early on in the disease there are not as many autoreactive $\mathrm{T}$ cells that destroy a patient's insulin-producing cells.

"The planned prevention trial is not dependent on new loci being found," said Ringler. He said the study will be run in conjunction with the Juvenile Diabetes Research Foundation Center for Beta Cell Therapy.

"They have a predictive technology and have put together a set of markers that identify patients at risk. It's coming to more formal testing," he said.

\section{REFERENCES}

1. Zhernakova, A. et al. Am. J. Hum. Genet; ; published online Oct. 24, 2007; doi:10.1086/522037

Contact: Bobby Koeleman, Department of Medical Genetics, University Medical Center Utrecht, Utrecht, The Netherlands

e-mail: b.p.c.koeleman@umcutrecht.nl

2. van Heel, D.A. et al. Nat. Genet. 39, 827-829 (2007)

3. Wellcome Trust Case Control Consortium. Nature 447, 661-68 (2007)

4. Yamanouchi, J. et al. Nat. Genet. 39, 329-337 (2007)

COMPANIES AND RESEARCH INSTITUTIONS MENTIONED

Genmab A/S (CSE:GEN), Copenhagen, Denmark

GlaxoSmithKline plc (LSE:GSK; NYSE:GSK), London, U.K.

Juvenile Diabetes Research Foundation Center for Beta Cell

Therapy, Brussels, Belgium

Tolerx Inc., Cambridge, Mass.

University Medical Center Utrecht, Utrecht, The Netherlands 